\title{
Incidência da Leishmaniose Tegumentar Americana no Centro-Sul de Mato Grosso, Brasil entre 2000 a 2019
}

A Leishmaniose Tegumentar Americana (LTA) é uma doença zoonótica negligenciada, que afeta a pele e as mucosas, causada por diferentes espécies do gênero Leishmania. O objetivo deste estudo foi analisar o perfil demográfico, clínico, geográfico e temporal dos casos de LTA registrados no período $2000-2019$, na secretaria de saúde do município de Cáceres/MT. A informação demográfica dos casos registrados foi analisada por: Perfil demográfico, Aspectos clínicos, Distribuição geográfica e temporal e Taxa de incidência anual. A maior prevalência encontrada foi em homens na faixa etária de 20 a 60 anos. Aproximadamente $81 \%$ dos casos eram de leishmaniose cutânea. As profissões mais afetadas foram os lavradores e trabalhadores braçais. A proporção entre os casos rurais e urbanos foi similar. Ao analisar o perfil demográfico apenas dos casos urbanos, os aposentados, donas do lar e estudantes foram o grupo mais afetado. A LTA foi amplamente distribuída, em diversos bairros do centro urbano da cidade. Concluímos que a LTA em Cáceres/MT afeta principalmente a população produtiva masculina. Contudo, nos casos urbanos a população mais afetada está formada por aposentados, donas do lar e estudantes sugerindo a possibilidade de eventuais surtos endêmicos.

\section{Incidence of American Tegumentary Leishmaniasis in the Center- South of Mato Grosso, Brazil between 2000 to 2019}

\begin{abstract}
American Tegumentary Leishmaniasis (ATL) is a neglected zoonotic disease that affects the skin and mucous membranes, caused by different species of the Leishmania genus. The aim of this study was to analyze the demographic, clinical, geographic and temporal profile of cases of ATL recorded in the period 20002019, at the health department of the municipality of Cáceres/MT. The demographic information of the registered cases was analyzed by: Demographic profile, Clinical aspects, Geographic and temporal distribution and Annual incidence rate. The highest prevalence found was in men aged 20 to 60 years. Approximately $81 \%$ of cases were cutaneous leishmaniasis. The most affected professions were lumberjacks and manual workers. The proportion between rural and urban cases was similar. When analyzing the demographic profile of only urban cases, retirees, homemakers and students were the most affected group. LTA was widely distributed in several neighborhoods in the city's urban center. We conclude that ATL in Cáceres/MT mainly affects the male productive population. However, in urban cases the most affected population is made up of retirees, housewives and students suggesting the possibility of possible endemic outbreaks.
\end{abstract}

Keywords: Leishmaniasis; Cutaneous; Incidence; Epidemiology; Prevalence.

Topic: Epidemiologia e Saúde Ambiental

Reviewed anonymously in the process of blind peer.
Received: 01/05/2020

Approved: 02/06/2020
Julianne Pachiega (iD

Universidade do Estado de Mato Grosso, Brasil http://lattes.cnpq.br/0846423790132609 http://orcid.org/0000-0002-9303-1898

jupachiega@gmail.com

Giovanna Maria Simioni Silva (iD)

Universidade do Estado de Mato Grosso, Brasil

http://lattes.cnpq.br/7327576212621799

http://orcid.org/0000-0002-6629-0026

giovannasimioni1996@gmail.com

Rose Margarethe Costa (iD

Universidade do Estado de Mato Grosso, Brasil

http://lattes.cnpq.br/6760907280992490

http://orcid.org/0000-0002-8740-8644

rosemargarethecosta@gmail.com

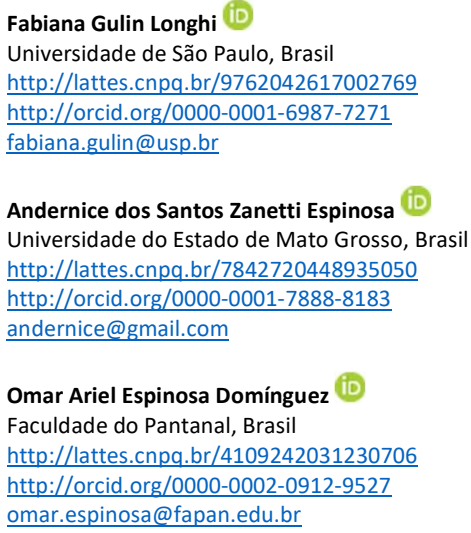

Referencing this:

PACHIEGA, J.; SILVA, G. M. S.; COSTA, R. M.; LONGHI, F. G.; ESPINOSA, A. S. Z.; DOMÍNGUEZ, O. A. E.. Incidência da Leishmaniose Tegumentar Americana no Centro-Sul de Mato Grosso, Brasil entre 2000 a 2019. Revista Ibero-Americana de Ciências Ambientais, v.11, n.4, p.126135, 2020. DOI: http://doi.org/10.6008/CBPC21796858.2020 .004 .0011 


\section{INTRODUÇÃO}

A Leishmaniose Tegumentar Americana (LTA) é uma doença zoonótica, que afeta a pele e as mucosas, causada por diferentes espécies de protozoários do gênero Leishmania (ROSS, 1903; GONTIJO et al., 2003). Esta doença apresenta diferentes manifestações clínicas, sendo as principais a leishmaniose cutânea (LC), leishmaniose cutânea disseminada (LCD), leishmaniose difusa (LD) e a leishmaniose mucocutânea (LMC) (GOTTO et al., 2010).

A LTA além de ser uma doença negligenciada, é considerada uma doença importante devido ao seu alto coeficiente de deteç̧ão e capacidade de produzir deformidades, dessa forma, a doença afeta principalmente indivíduos de baixo nível socioeconômico e o número de casos em áreas endêmicas têm aumentado significativamente nos últimos anos (COPELAND et al., 2015; ALVAR et al., 2012; MATHERS et al., 2007; GALGAMUWA et al., 2019).

Segundo dados da Organização Pan-Americana da Saúde (OPAS), nas Américas, no período de 20012017, um total de 940.396 casos novos de leishmaniose cutânea (LC) e mucosa (LM) foram reportados por 17 dos 18 países endêmicos, com uma média anual de 55.317 casos, sendo o Brasil responsável por 72,6\% dos casos (OPAS, 2019). No estado de Mato Grosso, entre 2001-2017 foram notificados através do Sistema de Informação de Agravos de Notificação (SINAN) 52.637 casos, ou seja, um 12,75\% dos casos notificados no país neste período (BRASIL, 2010).

Dados do Ministério da Saúde entre 1989 e 2008 revelaram um aumento significativo no número de casos confirmados no Centro-Oeste (BRASIL, 2010). Em Mato Grosso, a LTA é uma das mais importantes infecções dermatológicas, devido à sua alta incidência e relevante endemicidade, colocando o estado em segundo lugar em nível nacional de detecção de casos.

Apesar dos dados do ministério da saúde, poucos estudos foram realizados com o objetivo de apresentar a incidência da LTA dentre os municípios do estado de Mato Grosso. Contudo, um estudo que analisou casos no período 2001-2008 mostrou que municípios como Sinop e Sorriso, ambos localizados na zona norte do estado, apresentaram uma maior ocorrência de casos notificados. No entanto, outros municípios como Itanhingá, Santa Rita do Trivelato e Nova Santa Helena notificaram uma baixa quantidade de casos neste período de tempo (NOBRE et al., 2008). Outra análise de casos realizada no município de Chapada dos Guimarães reportou 153 casos notificados no período 2003-2013 (ARAÚJO et al., 2015).

A epidemiologia da LTA tradicionalmente foi definida como uma zoonose transmitida aos seres humanos quando eles entram em contato com os vetores nas florestas, embora este conceito vem sendo alterado com os resultados de novas pesquisas que apontam que essa doença está surgindo cada vez mais em zonas periurbanas e urbanas (BENíCIO et al., 2015). Por exemplo, uma série de casos analisados no período 2010-2014, constatou que não foram encontradas diferenças entre as zonas urbanas e rurais em relação a percentagem de distribuição de casos novos de LTA no Amazonas (TELES et al., 2019).

Em relação ao perfil demográfico dos pacientes afetados por LTA, de acordo com os dados registrados no SisLeish em 2017, 68,7\% (34.305) dos casos corresponderam ao sexo masculino sendo a faixa 
etária de $\geq 20-<50$ anos os mais afetados (OPAS, 2019). Em relação ao perfil ocupacional, está associado principalmente a uma alta incidência de atividades como mineração ou prospecção de ouro, agricultura, desmatamento para produção de gado e transformações ambientais relacionadas ao aumento da migração rural (NOBRES et al., 2013; BENÍCIO et al., 2015; ROCHA et al., 2015).

O município de Cáceres se localiza na região Centro-Sul de Mato Grosso e na microrregião do Alto Pantanal, sendo a atividade agropecuária a principal fonte econômica da cidade. O crescimento urbano e os tipos de atividades desenvolvidas na região podem estar relacionados com o número de casos de leishmaniose tegumentar americana registrados. Contudo, atualmente não existem dados publicados sobre o perfil epidemiológico da LTA nos casos registrados e notificados desta região. Por este motivo, este estudo teve como objetivo analisar o perfil demográfico, clínico, geográfico e temporal dos casos de LTA registrados no período 2000-2019, na secretaria de saúde do município de Cáceres/MT.

\section{METODOLOGIA}

\section{Desenho de estudo}

Trata-se de um estudo epidemiológico com base nos dados estatísticos dos casos registrados anualmente pela secretaria municipal de saúde do município de Cáceres/MT e que são notificados pelo Sistema de Informação de Agravos e Notificação (SINAN).

\section{Área de estudo}

O estudo foi desenvolvido no município de Cáceres situado no centro-sul de Mato Grosso, região centro-oeste do Brasil, integrando a microrregião do alto Pantanal e a mesorregião do centro-sul matogrossense, com uma área territorial de $24.796,8 \mathrm{~km}^{2}$. O principal bioma deste município é o Pantanal o qual recobre mais de $50 \%$ do território (COUTINHO et al., 1997). A população estimada, segundo o Instituto Brasileiro de Geografia e Estatística (IBGE) no ano de 2019 foi de aproximadamente 94.376 pessoas (Censo demográfico 2010).

\section{População de estudo e fontes de dados}

A população de estudo consiste em todos os casos (novos e antigos) de LTA registrados pela secretária de saúde e devidamente notificados pelo SINAN no município de Cáceres (Mato Grosso), desde o dia 1 de janeiro de 2000 até 31 de dezembro de 2019. A informação demográfica dos casos anuais de LTA registrados no município de Cáceres/MT, foram obtidos a partir de dados estatísticos da secretaria de saúde do município, enquanto que os dados do SINAN foram obtidos através do ministério da saúde. Devido a serem dados secundários, a aprovação do comitê de ética em pesquisa para estudos envolvendo seres humanos não foi necessária, de acordo com o Conselho Nacional de Saúde, resolução 510/2016. As estimativas da população anual de Cáceres/MT, para cada ano do período 2000-2019, foram obtidas através do Instituto Brasileiro de Geografia e Estatística (IBGE, 2010). 


\section{Variáveis}

Os dados foram analisados por: 1) Perfil demográfico (ano de registro, sexo, faixa etária e profissão); 2) Aspectos clínicos (tipo de caso e forma clínica); 3) Distribuição geográfica e temporal (área, distribuição dos casos segundo o bairro por quinquênio); 4) Taxa de incidência anual.

\section{Análise de dados}

Os dados de cada variável foram resumidos utilizando estatística descritiva, através de valores de porcentagens. Foi feita uma análise demográfica, clínica e de distribuição geográfica temporal dos dados gerais e outra apenas dos casos registrados em bairros da área urbana. Finalmente, um gráfico de regressão linear foi realizado utilizando as taxas de incidência anuais.

\section{RESULTADOS}

Em total a secretaria municipal de saúde do município de Cáceres registrou 675 casos durante o período de 2000 a 2019. Após realizar a busca no SINAN constatamos que durante o período de tempo de 2001 a 2017 foram notificados 536 casos. Para este mesmo período de tempo (2001 - 2017), a secretaria de saúde de Cáceres registrou 532 casos. Devido os registros estatísticos da secretaria de saúde municipal possuírem uma maior quantidade de informação, decidimos realizar as análises do estudo a partir desses dados secundários.

\section{Perfil demográfico}

Dos 675 casos registrados pela secretaria de saúde, 550 (78,3\%) eram do sexo masculino e 152 $(21,7 \%)$ do sexo feminino. Os adultos entre 21 a 60 anos foram o grupo mais afetados com $487(72,1 \%)$ casos registrados (Tabela 1). Entre as profissões mais afetadas se destacam: 107 (16,77\%) lavradores, 60 (9,40\%) aposentados, $58(9,10 \%)$ donas do lar, 54 (8,46\%) trabalhadores braçais, $46(7,21 \%)$ estudantes, 23 (3,60\%) serviços gerais, 21 (3,30\%) vaqueiros, 20 (3,13\%) militares, 17 (2,66\%) pedreiros, 11 (1,72\%) pecuaristas, 11 $(1,72 \%)$ motoristas, $10(1,57 \%)$ agricultores e $8(1,25 \%)$ pescadores.

\section{Aspectos clínicos}

Quando analisada a forma clínica da doença observamos que $550(81,4 \%)$ eram casos de leishmaniose cutânea, 119 (17,6\%) leishmaniose mucosa, 1 misto $(0,2 \%)$ e $5(0,8 \%)$ casos que não foram identificados. Em relação ao tipo de caso, $561(88,6 \%)$ eram casos novos e $77(11,4 \%)$ casos antigos (Tabela 1). Em total foram utilizadas aproximadamente 38.696 ampolas de antimoniato de meglumina para tratar os 647 pacientes e, nos 28 pacientes restantes, não foi informada a quantidade de ampolas administradas.

Tabela 1: Distribuição das características demográficas e clínicas dos casos registrados de leishmaniose tegumentar americana em Cáceres/MT de 2000 a 2019. 


\begin{tabular}{|c|c|c|c|c|c|c|c|c|c|c|c|c|c|c|c|c|c|c|c|c|c|}
\hline & $\begin{array}{c}2000 \\
n=86\end{array}$ & $\begin{array}{c}2001 \\
n=85\end{array}$ & $\begin{array}{c}2002 \\
n=51\end{array}$ & $\begin{array}{c}2003 \\
n=57\end{array}$ & $\begin{array}{c}2004 \\
n=33\end{array}$ & $\begin{array}{c}2005 \\
n=20\end{array}$ & $\begin{array}{c}2006 \\
n=19\end{array}$ & $\begin{array}{c}2007 \\
n=20\end{array}$ & $\begin{array}{c}2008 \\
n=25\end{array}$ & $\begin{array}{c}2009 \\
n=17\end{array}$ & $\begin{array}{c}2010 \\
n=20\end{array}$ & $\begin{array}{l}2011 \\
n=4\end{array}$ & $\begin{array}{c}2012 \\
n=11\end{array}$ & $\begin{array}{c}2013 \\
n=28\end{array}$ & $\begin{array}{c}2014 \\
n=41\end{array}$ & $\begin{array}{c}2015 \\
n=43\end{array}$ & $\begin{array}{c}2016 \\
n=16\end{array}$ & $\begin{array}{c}2017 \\
n=42\end{array}$ & $\begin{array}{c}2018 \\
n=20\end{array}$ & $\begin{array}{c}2019 \\
n=37\end{array}$ & $\begin{array}{c}\text { Casos } \\
\text { registrados } \\
n=675\end{array}$ \\
\hline & $\%$ & $\%$ & $\%$ & $\%$ & $\%$ & $\%$ & $\%$ & $\%$ & $\%$ & $\%$ & $\%$ & $\%$ & $\%$ & $\%$ & $\%$ & $\%$ & $\%$ & $\%$ & $\%$ & $\%$ & \\
\hline \multicolumn{22}{|l|}{ Descrição } \\
\hline Masculino & 75,5 & 82,3 & 90,2 & 87,8 & 87,8 & 70 & 78,9 & 75 & 80 & 70,5 & 90 & 50 & 90,9 & 64,3 & 82,9 & 62,8 & 75 & 71,4 & 90 & 78,4 & \\
\hline Feminino & 24,5 & 17,4 & 9,8 & 12,2 & 12,2 & 30 & 21,1 & 25 & 20 & 29,5 & 10 & 50 & 9,1 & 35,7 & 17,1 & 37,2 & 25 & 28,6 & 10 & 21,6 & \\
\hline \multicolumn{22}{|l|}{ Faixa Etária } \\
\hline$<10$ & 1,1 & 1,2 & 2 & 3,6 & 2,9 & - & 5,3 & - & - & - & - & - & - & - & 2,4 & 4,7 & - & 2,4 & 5 & 2,7 & \\
\hline $11-20$ & 13,9 & 9,4 & 5,9 & 10,7 & 2,9 & 20 & 10,5 & 15 & - & 5,9 & 5 & - & 27,3 & 7,1 & 12,2 & 11,7 & - & 11,9 & 5 & 8,1 & \\
\hline $21-30$ & 12,8 & 22,4 & 23,1 & 19,7 & 20,5 & 10 & 10,5 & 5 & 16 & 35,3 & 10 & 25 & 9,1 & - & 12,2 & 28 & 25 & 11,9 & 15 & 19 & \\
\hline $31-40$ & 16,3 & 28,2 & 25,4 & 17,9 & 14,8 & 25 & 15,8 & 15 & 24 & 23,6 & 30 & - & 18,2 & 21,4 & 24,4 & 13,9 & 18,8 & 21,4 & 20 & 21,6 & \\
\hline $41-50$ & 18,6 & 21,2 & 16,2 & 21,5 & 26,5 & 30 & 5,3 & 25 & 12 & 11,8 & 25 & - & 9,1 & 21,4 & 21,9 & 13,9 & 18,8 & 14,3 & 25 & 13,5 & \\
\hline $51-60$ & 15,1 & 9,4 & 17,6 & 9 & 17,6 & 10 & 36,9 & 5 & 32 & 5,9 & 15 & 25 & - & 21,4 & 19,5 & 13,9 & 25 & 23,8 & 5 & 21,6 & \\
\hline $61-70$ & 12,8 & 2,3 & 7,8 & 10,7 & 5,9 & 5 & 10,5 & 20 & 12 & 17,6 & 5 & - & 18,2 & 17,9 & 2,4 & 4,7 & 12,5 & 7,2 & 10 & 8,1 & \\
\hline $71-80$ & 9,3 & 1,2 & - & 7,1 & 8,9 & - & 5,3 & 15 & 4 & - & 10 & - & 9,1 & 10,7 & 2,4 & 9,3 & - & 4,8 & 15 & 5,4 & \\
\hline $81-90$ & - & 4,7 & 2 & - & - & - & - & - & - & - & - & 50 & - & - & 2,4 & - & - & 2,4 & - & - & \\
\hline $91-100$ & - & - & - & - & - & - & - & - & - & - & - & - & - & - & - & - & - & - & - & - & \\
\hline $100-105$ & - & - & - & - & - & - & - & - & - & - & - & - & 9,1 & - & - & - & - & - & - & - & \\
\hline \multicolumn{22}{|l|}{ Zona } \\
\hline Urbana & 57 & 36,5 & 51,8 & 48,2 & 11,8 & 15 & 40 & 42 & 48 & 5,8 & 50 & 100 & 27,3 & 57,1 & 46,4 & 34,9 & 43,8 & 42,9 & 80 & 64,8 & \\
\hline Rural & 41,8 & 63,5 & 41,2 & 48,2 & 79,4 & 55 & 55 & 48 & 52 & 82,5 & 45 & - & 54,5 & 39,3 & 51,2 & 60,4 & 56,2 & 57,1 & 15 & 35,2 & \\
\hline N.i. & 1,2 & - & - & 3,6 & 8,8 & 30 & 5 & 10 & - & 11,7 & 5 & - & 18,2 & 3,6 & 2,4 & 4,7 & - & 5 & 5 & - & \\
\hline \multicolumn{22}{|l|}{ Forma Clínica } \\
\hline Cutânea & 81,4 & 80 & 80,4 & 76,4 & 52,9 & 88,9 & 88,9 & 40 & 72 & 100 & 65 & 75 & 81,8 & 85,2 & 90,2 & 93 & 100 & 90,5 & 90 & 91,9 & \\
\hline Mucosa & 19,6 & 20 & 19,6 & 23,6 & 44,1 & 11,1 & 11,1 & 50 & 28 & - & 25 & 25 & 18,2 & 14,8 & 9,8 & 7 & - & 7,1 & 10 & 8,1 & \\
\hline Misto & - & - & - & - & - & - & - & 5 & - & - & - & - & - & - & - & - & - & - & - & - & \\
\hline N.I. & - & - & - & - & 2,9 & - & - & 5 & - & - & 10 & - & - & - & - & - & - & 2,4 & - & - & \\
\hline \multicolumn{22}{|l|}{ Tipo de Caso } \\
\hline Novo & 64 & 82,3 & 82,3 & 91,1 & 97,1 & 90 & 94,7 & 95 & 92 & 100 & 100 & 100 & 81,8 & 92,2 & 100 & 93 & 100 & 92,9 & 100 & 100 & \\
\hline Velho & 36 & 17,7 & 17,7 & 8,9 & 2,9 & 10 & 5,3 & 5 & 8 & - & - & - & 18,2 & 7,8 & - & 7 & - & 7,1 & - & - & \\
\hline
\end{tabular}

\section{Distribuição geográfica e temporal}

Segundo a classificação por área, 350 (52\%) casos foram registrados em áreas rurais, 301 (44,5\%) em bairros da área urbana da cidade de Cáceres e em 24 (3,5\%) casos não foi identificada a localidade. Os anos de notificações foram agrupados em quatro quinquênios (2000-2004, 2005-2009, 2010-2014 e 2015-2019) sendo o quinquênio $2000-2004$ o período que apresentou a maior quantidade de casos registados (46,2\%) seguido do período 2015-2019 (23,4\%). Gráficos da distribuição dos casos segundo o mês de registro foram realizados para cada quinquênio (figura 1 ).

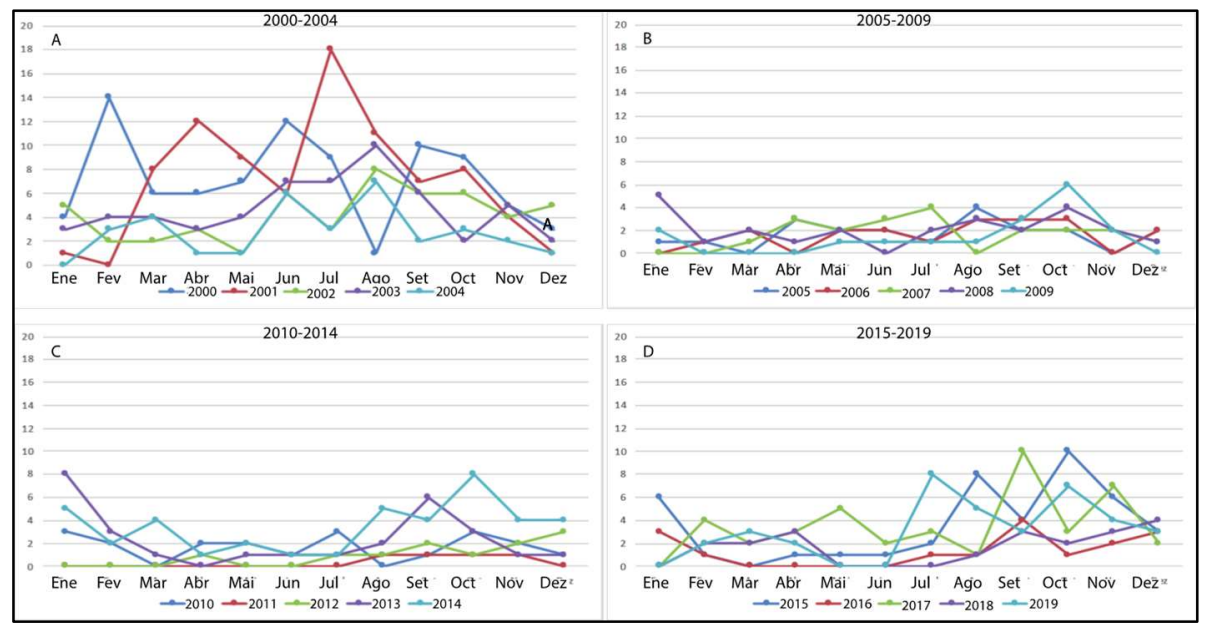

Figura 1: Distribuição dos casos de LTA registrados pela secretaria de saúde de Cáceres/MT, no período 2000 - 2019, segundo o quinquênio de registro. A) 2000 - 2004; B) 2005 - 2009; C) 2010 - 2014; D) 2015 - 2019.

Após distribuir os casos por meses observamos que o inverno (julho-setembro) é a estação do ano 
que mais casos foram registrados 223 (33\%) seguido pela primavera com 184 casos (27,3\%). Desta forma o semestre do ano compreendido entre os meses de julho - dezembro, foram registrados 407 casos (60,3\%) nos vinte anos estudados. Por outro lado, nas estações de verão e outono foram registrados 130 casos (19,3 $\%)$ e 138 casos $(20,4 \%)$ respectivamente, ou seja, 262 casos $(39,7 \%)$ no primeiro semestre do ano.

\section{Distribuição geográfica, demográfica e temporal dos casos urbanos}

Através do aplicativo de Google Maps, os 301 casos urbanos foram localizados em diferentes bairros da área urbana da cidade de Cáceres. Os bairros que apresentam maior quantidade de casos foram: Cavalhada com 52 casos (17,3\%), Jardim Padre Paulo com 20 (6,6\%), Vila Mariana 11 (3,6\%), e Jardim do Trevo, Jardim Guanabara, Santa Cruz e Santa Isabel com 10 (3,3\%) casos cada um (Figura 2).

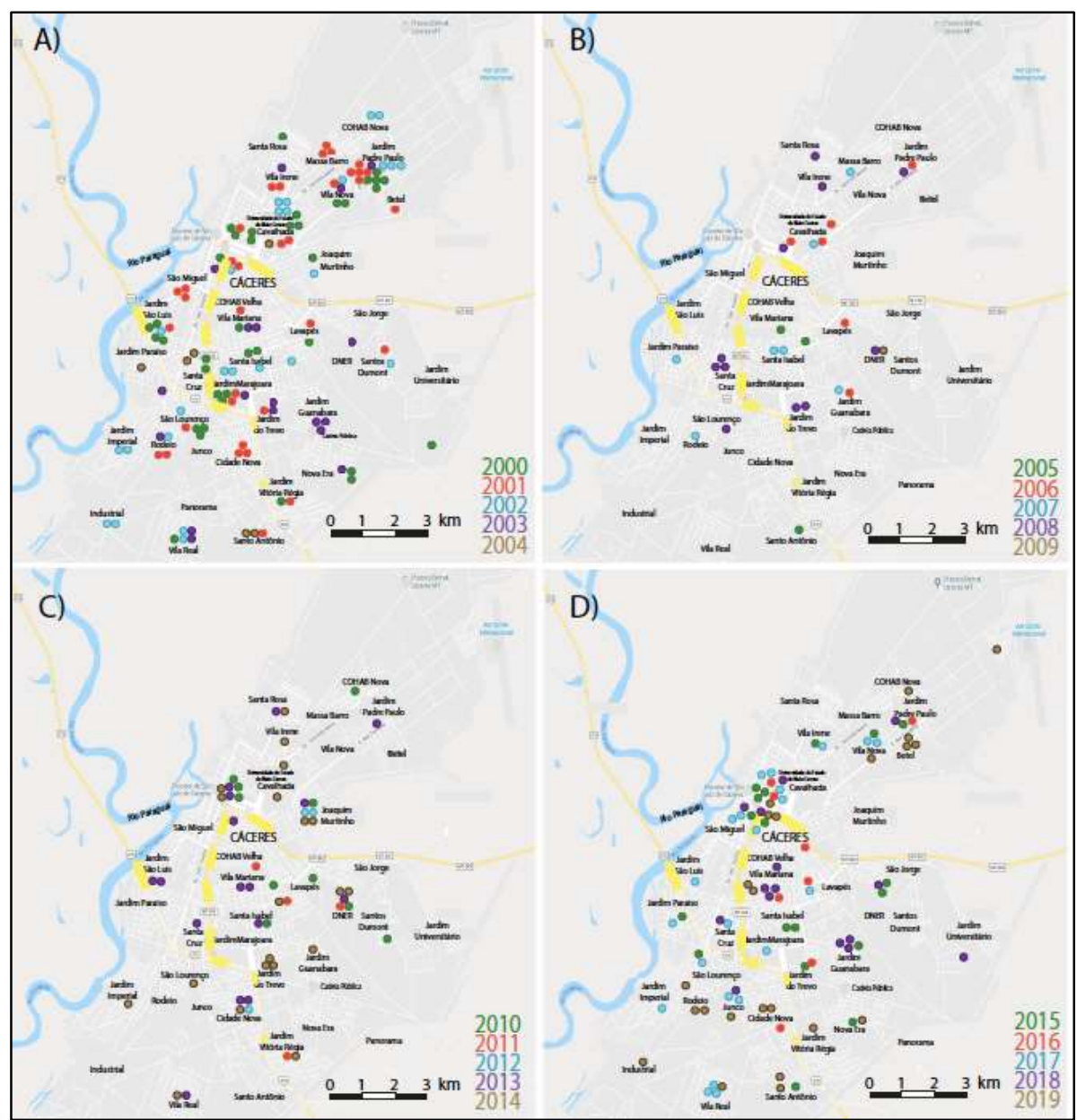

Figura 2: Distribuição dos casos urbanos de LTA do município de Cáceres/MT por quinquênio, segundo o bairro de registro. A) 2000 a 2004B) 2005 a 2009; C) 2010 a 2014; D) 2015 a 2019.

O perfil demográfico destes 301 casos, resultou similar ao perfil geral do município, sendo o sexo masculino, os adultos de 20 a 60 anos os mais afetados com $77,8 \%$ e $68,4 \%$ dos casos respectivamente. Em relação à profissão observamos que o grupo que apresentou uma maior quantidade de casos foram os aposentados, com $14 \%$ dos casos. O segundo grupo mais afetado foram as donas do lar com 9,3\% e estudantes e lavradores ambos com $7 \%$. Outras profissões afetadas foram os trabalhadores braçais com $4,21 \%$ e serviços gerais e pedreiros com $3,83 \%$ cada um. 
Os casos urbanos foram separados segundo o ano de registro, e agrupados em quatro quinquênios previamente definidos. Utilizando um mapa da cidade de Cáceres/MT, foi realizada uma distribuição dos casos para cada período de tempo (Figura 2). O primeiro quinquênio (2000-2004) concentra a maior quantidade de casos 139 (46,2\%), nos dois períodos seguintes houve uma diminuição de casos com 31 $(10,3 \%)$ e 57 (19\%), respectivamente. No último quinquênio registrou-se um aumento para $74(24,5)$ casos.

\section{Taxa de incidência anual}

O gráfico da taxa de incidência anual, demonstra uma alta incidência da LTA no município de Cáceres, a qual foi diminuindo para o fim do primeiro quinquênio estudado. No segundo quinquênio houve uma diminuição constante da taxa de incidência. Contudo, nos últimos oito anos foi registrado um aumento da incidência da LTA com queda apenas nos anos 2016 e 2018 (Figura 3).

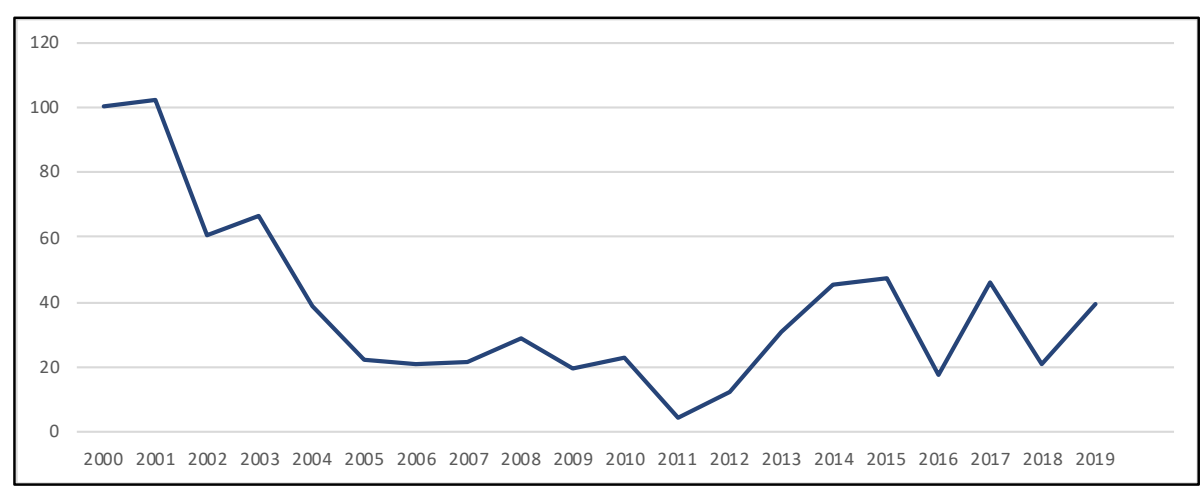

Figura 3: Taxa de incidência de leishmaniose tegumentar americana por 100000 habitantes, Cáceres/MT, 2000-2019.

\section{DISCUSSÃO}

De modo geral, o perfil demográfico dos casos registrados no município de Cáceres-MT, é semelhante ao reportado no Informe Epidemiológico das Américas da OPAS (SisLeish 2017). Nesse sentido, houve uma maior proporção nos pacientes do sexo masculino, e aproximadamente $72 \%$ dos pacientes acometidos correspondiam a faixa etária entre 20-60, sendo a leishmaniose cutânea (LC) a principal forma clínica, embora, uma porcentagem importante de casos de leishmaniose mucocutânea (LMC) foi registrada. Este padrão também foi observado em outros estudos similares realizado no estado do Amazonas e em diversos municípios de Mato Grosso (NOBRES et al., 2013; TELES et al., 2019). É importante destacar que nesse Informe Epidemiológico das Américas da OPAS (OPAS, 2019), foi reportado o grupo de idades entre 20 a 50 anos como o mais afetado, contudo, no nosso estudo decidimos ampliar este grupo etário devido à idade de aposentadoria no Brasil ser de 62 e 65 para mulheres e homens, respectivamente. Por este motivo, a LTA representa um problema de saúde pública que pode levar a agravos físicos, econômicos e morais na população produtiva masculina do município de Cáceres/MT.

O perfil ocupacional dos pacientes com LTA, registrados em Cáceres/MT, está associado principalmente a uma alta incidência de atividades de trabalho pesado como desmatamento, agricultura e produção de gado, além do resultado das transformações ambientais relacionadas ao aumento da migração rural, como foi relatado em estudos anteriores (NOBRES et al., 2013; BENÍCIO et al., 2015; ROCHA et al., 
2015). Considerando que a região de Cáceres/MT se caracteriza por ser um setor dedicado ao agronegócio e atividades de desmatamento como principais atividades econômicas, o ofício de lavrador e trabalhador braçal, resultam em profissões que poderiam representar um fator de risco na infecção com LTA.

Como podemos observar no nosso estudo, houve proporções similares de pacientes registrados tanto em áreas rurais quanto no centro urbano de Cáceres/MT. Isso pode ser devido às mudanças ambientais relacionadas à migração, urbanização e aspectos socioeconômicos relacionados às profissões de risco como lavradores e trabalhador braçais (na roça).

É importante destacar que entre os casos registrados com LTA no centro urbano de Cáceres/MT, aproximadamente um terço (30\%) são aposentados, donas do lar e estudantes. Este grupo está composto por pacientes que teoricamente não exercem atividade remunerada, portanto, são um grupo que passam mais tempo no centro urbano da cidade. Esta porcentagem de casos registrados nessa população, nos leva a considerar que eventuais surtos endêmicos possam estar acontecendo no centro urbano da cidade como foi demonstrando em outras áreas endêmicas do Brasil (BENÍCIO et al., 2015; CARNEIRO et al., 2018).

A LTA no município de Cáceres/MT mostra um padrão sazonal com predomínio de casos no segundo semestre de cada ano, o qual se caracteriza por ser mais quentes e seco na região. Este padrão de variação sazonal, difere de outro estudo realizado em zonas endêmicas do Amazonas, onde foi demonstrado que este padrão estava relacionado com os períodos de chuva (GUERRA et al., 2015). Outro estudo demonstra um aumento da população de flebotomíneos durante o período chuvoso (SARAIVA et al., 2017). Devido ao fato de não observar diferencias entre a quantidade de os casos urbanos e rurais registrados, não podemos descartar a possibilidade de que os vetores possam ter se adaptado a novos ambientes peridomiciliares, já que as populações de flebotomíneos na região ainda é desconhecida. Por este motivo, se recomenda a realização de estudos que possam identificar os possíveis vetores desta doença no município de Cáceres/MT.

Devido à LTA ser uma zoonose, devemos considerar a interação dos seres humanos com os diferentes tipos de animais que atuam como reservatórios das espécies de Leishmania. Nos últimos anos a cidade de Cáceres se caracterizou pelo aparecimento de grandes mamíferos (onças) tanto na periferia quanto no centro da cidade. Por outro lado, por estar próxima da Serra do Mangaval, Ponta do Morro e às margens do rio Paraguai, é comum observar pequenos e grandes mamíferos (gambá, quatis, capivaras, cotia e pequenos roedores). Alguns destes animais foram descritos anteriormente como reservatórios de espécies de Leishmania (ARIAS et al., 1981; SILVEIRA et al., 1991; MORENO et al., 2020; RICHINI-PEREIRA et al., 2014). Sendo a época seca e quente do ano a que mais casos de LTA registrou, resulta importante estudar a interação dos reservatórios com os seres humanos durante este período, já que a transmissão da doença pode estar relacionada à biologia destes animais ou às alterações que o ser humano vem realizando no seu ambiente.

Em relação à taxa de incidência, observamos uma alta taxa entre os primeiros cinco anos de estudo e foi diminuindo nos anos seguintes. Por outro lado, nos últimos cinco anos (2014-2019) foi registrado um aumento de casos, inclusive maior que o reportado nos dez anos anteriores (2005-2014). Esta tendência nos 
leva a considerar que enquanto medidas de controles do vetor e de prevenção da doença não sejam tomadas, o número de casos registrados no município de Cáceres/MT poderá se manter nos próximos anos ou inclusive registrar um aumento considerável.

\section{CONCLUSÕES}

Podemos concluir que embora o perfil laboral permaneceu semelhante ao longo dos vinte anos estudados, esta característica muda quando analisados apenas os casos da zona urbana de Cáceres/MT, sugerindo a possibilidade de eventuais surtos endêmicos. Por esse motivo, sugere-se o desenvolvimento de pesquisas com o objetivo de estudar possíveis reservatórios e vetores na região, afim de tomar medidas de controles que permitam diminuir os números de casos registrados no futuro. Sugere-se ainda, que ações estratégicas sejam implementadas entre trabalhadores que exercem atividades consideradas de risco na infecção com LTA, especificamente lavradores e trabalhadores da roça. Os resultados apresentados neste estudo visam orientar a secretaria de saúde na formulação de medidas de prevenção nas áreas e populações mais vulneráveis.

\section{REFERÊNCIAS}

ALVAR, J.; VÉLEZ, I. D.; BERN, C.; HERRERO, M.; DESJEUX, P.; CANO, J.; JANNIN, J.; DEN BOER, M.. WHO Leishmaniasis Control Team Leishmaniasis worldwide and global estimates of its incidence. PLoS One, v.7, n.5, e35671, 2012.

ARAÚJO, A. F.; CRUZ, C. G.; SILVA, D. A.; MARQUES, L. F.; FERREIRA, M. R.; CAVICHIOLI, A. A. B.. Leishmanioses no município de Chapada dos Guimarães. Coorte: Revista Científica do Hospital Santa Rosa, n.5, p.44-9, 2015.

ARIAS, J. R.; NAIF, R. D.; MILES, M. A.; SOUZA, A. A.. The opossum, Didelphis marsupialis (Marsupialia: Didelphidae), as a reservoir host of Leishmania braziliensis guyanensis in the Amazon Basin of Brazil. Transactions of the Royal Society of Tropical Medicine and Hygiene, v.75, n.4, p.53741, 1981.

BENÍCIO, E.; CORDEIRO, M.; MONTEIRO, H.; MOURA, M. A S.; OLIVEIR, C.; GADELHA, E. P. N.; CHRUSCIAK-TALHARI, A.; TALHARI, C.; FERREIRA, L. C. L.; MIRA, M. T.; MACHADO, P. R. S.; TALHARI, S.; SCHRIEFER, A.. Sustained presence of leishmaniasis in urban Manaus, the largest human settlement in the Amazon. American Journal of Tropical Medicine and Hygiene, v.93, n.6, p.1208-13, 2015.

BRASIL. Ministério da Saúde. Manual de Vigilância da Leishmaniose Tegumentar Americana. 2 ed. Brasília: Ministério da Saúde, 2010.

CARNEIRO, F. R. O.; AMIN, G. A.; CRUZ, L. B. P.; DAHER, B. A. Urban American cutaneous leishmaniasis. Anais Brasileiros de Dermatologia, v.93, n.1, p.56-158, 2018.

COPELAND, N. K.; ARONSON, N. E.. Leishmaniasis: treatment updates and clinical practice guidelines review. Current Opinion in Infectious Disease, v.28, n.5, p.426-37, 2015.
COUTINHO, M.; CAMPOS, Z.; MOURÃO, G.; MAURO, R.. Aspectos ecológicos dos vertebrados terrestres e semiaquáticos no Pantanal. In: BRASIL. Ministério do Meio Ambiente, dos Recursos Hídricos e da Amazônia Legal. Plano de conservação da Bacia do Alto Paraguai

(Pantanal) - PCBAP: diagnóstico dos meios físicos e bióticos: meio biótico. Brasília, 1997. p.183-322.

GALGAMUWA, L. S.; DHARMARATNE, S. D.; IDDAWELA, D. Leishmaniasis in Sri Lanka: spatial distribution and seasonal variations from 2009 to 2016. Parasites \& Vectors, v.11, n.1, p.60, 2018.

GONTIJO, B.; CARVALHO, M. D.. Leishmaniose tegumentar americana. Revista da Sociedade Brasileira de Medicina Tropical, v.36, n.1, p.71-80, 2003

GOTO, H.; LINDOSO, J. A.. Current diagnosis and treatment of cutaneous and mucocutaneous leishmaniasis. Expert Review of Anti-infective Therapy, v.8, n.4, p.419-33, 2010.

GUERRA, J. A.; MACIEL, M. G.; GUERRA, M. V.; TALHARI, A. C.; PRESTES, S. R.; FERNANDES, M. A.; CRUZ, A. M.; MARTINS, A.; COELHO, L. I.; ROMERO, G. A.; BARBOSA, M. D.. Tegumentary leishmaniasis in the State of Amazonas: what have we learned and what do we need?. Revista da Sociedade Brasileira de Medicina Tropical, v.48, n.1, p.12-9, 2015.

IBGE. Instituto Brasileiro de Geografia e Estatística. Censo demográfico 2010. Brasília: IBGE, 2010.

MATHERS, C. D.; EZZATI, M.; LOPEZ, A. D.. Measuring the burden of neglected tropical diseases: the global burden of disease framework. PLoS Neglected Tropical Disease, v.1, n.2, p.e114, 2007 
MORENO, E. S.; SABIONI, L. A.; SEIXAS, M. M. M.; SOUZA FILHO, J. A.; MARCELINO, A. P.; SHIMABUKURO, P. H. F.. Evidence of a sylvatic enzootic cycle of Leishmania infantum in the State of Amapá, Brazil. Revista da Sociedad Brasileira de Medicina Tropical, v.53, p.e20190169, 2020.

NOBRES, E. S.; SOUZA, L. A.; RODRIGUES, D. J.. Incidência de leishmaniose tegumentar americana no norte de Mato Grosso entre 2001 e 2008. Acta Amazônica, v.43, n.3, p.297303, 2013.

OPAS. Organización Panamericana de la Salud. Leishmaniasis: informe epidemiológico de las Américas. Informe de Leishmaniasis, 2019.

RICHINI-PEREIRA, V. B.; MARSON, P. M.; HAYASAKA, E. Y.; VICTORIA, C.; SILVA, R. C.; LANGONI, H.. Molecular detection of Leishmania spp. in road-killed wild mammals in the Central Western area of the State of São Paulo, Brazil. Journal of Venomous Animals and Toxins including Tropical Diseases, v.20, n.27, p.1-7, 2014.

ROCHA, T. J.; BARBOSA, A. C.; SANTANA, E. P.; CALHEIROS, C. M.. Aspectos epidemiológicos dos casos humanos confirmados de leishmaniose tegumentar americana no
Estado de Alagoas, Brasil. Revista Pan-Amazônica de Saúde, v.6, n.4, p.49-54, 2015.

ROSS, R.. Note on the bodies recently described by leishman and Donovan. British Medical Journal, v.2, n.2237, p.1261-2. 1903

SARAIVA, L.; LEITE, C. G.; LIMA, A. C.; CARVALHO, L. O.; PEREIRA, A. A.; RUGANI, J. M.; REGO, F. D.; GONTIJO, C. M.; ANDRADE FILHO, J. D.. Seasonality of sand flies (Diptera: Psychodidae) and Leishmania DNA detection in vector species in an area with endemic visceral leishmaniasis. Memórias do Instituto Oswaldo Cruz, v.112, n.4, p.309-18, 2017.

SILVEIRA, F. T.; LAINSON, R.; SHAW, J. J.; BRAGA, R. R.; ISHIKAWA, E. E.; SOUZA, A. A.. Cutaneous leishmaniasis in Amazon region: isolation of Leishmania (Viannia) lainsoni from the rodent Agouti paca (Rodentia: Dasyproctidae), in the Pará State, Brazil. Revista do Instituto de Medicina Tropical de Sao Paulo, v.33, n.1, p.18-22, 1991.

TELES, G. D. C.; FONSECA, F. R.; GONÇALVES, M. J. F.. American Tegumentary Leishmaniasis in the Brazilian Amazon from 2010 to 2014. Revista do Instituto de Medicina Tropical de Sao Paulo, v.61, p.e22, 2019.

A CBPC - Companhia Brasileira de Produção Científica (CNPJ: 11.221.422/0001-03) detém os direitos materiais desta publicação. Os direitos referem-se à publicação do trabalho em qualquer parte do mundo, incluindo os direitos às renovações, expansões e disseminações da contribuição, bem como outros direitos subsidiários. Todos os trabalhos publicados eletronicamente poderão posteriormente ser publicados em coletâneas impressas sob coordenação da Sustenere Publishing, da Companhia Brasileira de Produção Científica e seus parceiros autorizados. Os (as) autores (as) preservam os direitos autorais, mas não têm permissão para a publicação da contribuição em outro meio, impresso ou digital, em português ou em tradução. 